\title{
Discussion on applications of computer network technology in electronics and information engineering
}

\author{
Ruxin $\operatorname{Tan}^{1,}$ a \\ ${ }^{1}$ Department of Information Science and Engineering ,Southeast University,Nanjing 210000,China \\ a594720299@qq.com
}

\begin{abstract}
Keywords: computer network technology; electronics and information engineering
Abstract. With the rapid development of the era, Internet information technology has found extensive applications, thus playing an important role in improving people' s living standards. This paper conducts a basic analysis of applications of computer network technology in electronics and information engineering.
\end{abstract}

\section{Introduction}

Despite of rapid development China has achieved in terms of electronics and information technology, there is still a wide gap between China and developed countries. In order to boost development of China's electronics and information technology, it is imperative to combine computer network technology with electronics and information technology to conduct an in-depth research of electronics and information engineering.

\section{Computer network technology, electronics and information engineering}

\section{A. Computer network technology}

Computer network technology is a combination of communications technology and computer technology. During its applications, users should abide by certain network protocol and better transmit information through connection of some independent and scattered computer equipment. [1] Optical fibers, electric cables and twisted pairs are all optimal tools for information sending. The computer network has super-strong hardware equipment. Through resource sharing, some information resources can be collected and processed. After that, information, data, pictures and texts can be sent through the computer. With a high degree of safety, computer network technology has been widely-used in daily life. With the rapid development of society and the constant improvement of China's technology level, computer network technology has become an indispensable part of people's life.

B. Definition of electronics and information engineering

Electronics and information engineering includes not only collection and processing of information data, but also establishment of a reasonable electronic equipment information system for applications. In terms of the current stage of development level, electronics and information engineering has brought more convenient and comfortable services for people, greatly improving people's living standards. [2] Some commonly-used mobile equipment, such as mobile phones, iPads and notebooks, all contain electronics and information engineering technology. Electronics and information engineering technology is a huge system, and has contributed to rapid development of electronics and information engineering technology.

C. Characteristics of electronics and information engineering

During the application process, electronics and information engineering has the following characteristics: 1) Convenience: Electronics and information engineering can process orders sent out through the equipment, thus facilitating people's daily use. Besides, electronics and information engineering technology is fast and efficient in information processing. Convenience of electronics and information engineering is a critical reason for its popularity among people. 2) Accuracy: Electronics and information engineering is accurate in processing. Through relevant equipment, it can process relevant information, greatly increasing its accuracy. 3) Wide coverage: The major 
function of electronics and information engineering is information processing and applications. Along with constant development of the era, information resources of various trades and industries have become increasingly important. Electronics and information engineering has an edge in information sending. This is another reason for its wide applications. [3]

\section{Applications of computer network technology in electronics and information engineering}

Through electronics and information engineering, computer network technology can better send information and achieve resource sharing. Along with constant development of society, people's living standards are also improving. Applications of electronics and information engineering in daily life have drawn wide attention. Computer network technology can better collect, analyze and process information. With corresponding confidentiality measures, it can guarantee safety of communications equipment. Therefore, applications of computer network technology in electronics and information engineering can boost development of the latter. [4]

\section{A. Information sending}

The information era has witnessed explosive growth of information. Under the era background, electronics and information engineering should improve the application efficiency of computer network technology to facilitate people's information sending. Computer technology can guarantee safety and stability of information sending. It plays an important role in electronics and information engineering. Constant innovation of computer network technology, electronics and information engineering can better promote development of the current society.

\section{B. Information receiving}

During the application process of electronics and information engineering, nodes are often used to collect and receive some data. Through combination of computer network technology, electronics and information engineering, information can keep on providing, thus guaranteeing rapid and efficient information receiving. During the receiving process, computer network technology mainly does two things. First, it classified different information collected. Second, it conducts encrypted processing of information received to avoid information from being let out. For example, it can encrypt user information while users purchase or browse some items on the Internet. With computer network technology, information will be encrypted before being sent out, thus greatly increasing the safety of information receiving.

C. Information processing

During applications of network technology, information processing is an important task and plays an important role in electronics and information engineering. With electronics and information engineering, information processing can be more accurate, thus guaranteeing validity of information. [5] Besides, during the information processing process, some unknown risks and unpredictable factors might occur, bringing dangers to the whole network system. If some efficient firewalls can be used during information processing of computer network technology, probability of attack from external factors can be reduced, some negative technology can be further improved and loopholes of computer network technology can be immediately remedied. In this way, safety of information processing can be guaranteed and a more safe and reliable network environment can be created.

D. Electronic equipment

Development of electronics and information engineering cannot be separated from applications of some electronic equipment. In order to better combine the two, it is necessary to share information among electronic equipment through computer network technology. In this way, negative problems caused during information processing can be avoided, and electronic equipment can achieve greater development. Relevant information and materials can be collected through computer network technology. Through the test system, whether the data can be normally operated can be judged. In other words, computer network can contribute to information extraction and refinement of electronic equipment. Through constant operations of the information system, it can be seen that the working system and the communications system are of equal importance during the computer network application process. 
In order to promote rapid development of the market economy, applications of electronic equipment are also reflected in the following aspects. 1) Communication arteries: In the wide area network communication arteries, electronic information engineering can connect network communication ports of various users. 2) Communications media: Use computer network technology for information sending. 3) Web browser: Computer network technology can help read some information documents on the Internet. Through the browser, it can better check some necessary information.

\section{Safety of electronics and information engineering}

As the era moves ahead, computer network technology has found increasing applications. Meanwhile, it is faced with some problems as its applications in electronics and information engineering. These problems are diversified and might influence development of various fields. Professionals should immediately find out causes of these problems and provide reasonable solutions.

The above dangerous factors are generated through loopholes of network transmission routes and network information systems. Some criminals attack electronics and information engineering based on these loopholes. This not only damages interests of users, but also brings irreparable consequences to the whole electronics and information engineering through information sending. [6] Therefore, relevant technicians should get an in-depth understanding of computer network technology so as to isolate various risks. Even if the computer network still has problems, the losses thus incurred can be minimized. Besides, firewall technology can be used to prevent occurrence of serious consequences and guarantee safe network use.

\section{Conclusions}

Computer network technology is widely-used and popular in electronics and information engineering. During applications of computer network technology and in electronics and information engineering, it is necessary to obtain an in-depth understanding of electronics and information engineering to give full play to the role of electronics and information engineering in information resource transmission, sending and receiving. Information processing based on electronics and information engineering can provide a more efficient and scientific information technology for people, thus promoting better development of electronics and information engineering. This paper conducts a simple analysis of applications of computer network technology in electronics and information engineering. There are many limits of this research. The author hopes that more research can be conducted in this field to promote applications of computer network technology in electronics and information engineering.

\section{References:}

[1] FAN Xisong \& ZHANG Yong. A preliminary study of applications of computer network technology to electronics and information engineering[J]. Science and Technology \& Innovation, 2014, 22: 152 154.

[2] SHI Yuanfang. Application of computer network technology in electronics and information engineering[J]. Journal of Huaihai Institute of Technology(Natural Sciences Edition), 2015, 01: $45 \sim 47$.

[3] WANG Chong. Application of computer network technology in electronics and information engineering[J]. Journal of Chifeng College (Natural Sciences Edition), 2015, 14: 20 22.

[4] DENG Junjian. Application of computer network technology in electronics and information engineering[J]. Electronic Techniques and Software Engineering, 2014, 16: 208. 
[5] QIANG Xiaolong. Application of computer network technology in electronics and information engineering[J]. Journal of Chifeng College (Natural Sciences Edition), 2016, 01: 55-56.

[6] ZHU Hongnian. Discussion on application of computer network technology in electronics and information engineering[J]. Information \& Communications, 2016, 01: 167 168. 